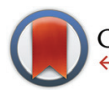

CrossMark

\& click for updates

Cite this: Food Funct., 2016, 7, 4432

Received 10th May 2016

Accepted 6th September 2016

DOI: $10.1039 / \mathrm{c} 6 f \circ 00685 \mathrm{j}$

www.rsc.org/foodfunction

\title{
Piceatannol is superior to resveratrol in promoting neural stem cell differentiation into astrocytes
}

\author{
Daisuke Arai, ${ }^{\text {a }}$ Ryousuke Kataoka, ${ }^{\mathrm{b}}$ Satoshi Otsuka, ${ }^{\mathrm{b}}$ Midori Kawamura, ${ }^{\mathrm{b}}$ \\ Hiroko Maruki-Uchida, ${ }^{c}$ Masahiko Sai, ${ }^{c}$ Tatsuhiko Ito ${ }^{c}$ and Yoichi Nakao*a,b
}

Piceatannol (3, 3', 4',5-trans-tetrahydroxystilbene) is a polyphenolic compound abundant in the seeds of passion fruit (Passiflora edulis). Piceatannol is an analogue of resveratrol (3,4',5-trans-trihydroxystilbene) and shares the structural motif and biological activities such as activation of SIRT1. Several studies have shown that piceatannol is more potent than resveratrol. In this study, we examined the effects of piceatannol on neural stem cell differentiation into astrocytes compared with those of resveratrol. At a concentration of $2.5 \mu \mathrm{M}$, piceatannol promoted astrocyte differentiation, while resveratrol had no effect at this concentration. Furthermore, we found that oral administration of piceatannol increased the number of astrocytes in the brains of adult mice, while resveratrol administration showed no effects. These results suggest that piceatannol has a superior effect to resveratrol in promoting astrocyte differentiation.

\section{Introduction}

Piceatannol $\left(3,3^{\prime}, 4^{\prime}, 5\right.$-trans-tetrahydroxystilbene) is a natural polyphenolic compound found in a limited number of plants, such as grapes, peanuts, Japanese knotweed, and some berries, and is particularly abundant in passion fruit (Passiflora edulis) seeds. ${ }^{1}$ Piceatannol is a $3^{\prime}$-hydroxylated analogue of resveratrol (3,4',5-trans-trihydroxystilbene). Both piceatannol and resveratrol have been reported to have antioxidative, anti-diabetic, anti-inflammatory, and anti-cancer effects. $^{2-10}$ Furthermore, transcriptional activation of the SIRT1 gene and/or enzymatic activation of the SIRT1 protein promote the activity of SIRT1, an NAD-dependent histone deacetylase. ${ }^{11-14}$ These biological activities are dependent on the similar structures of these molecules (Fig. 1); however, interestingly, previous studies have shown that piceatannol is more potent than resveratrol in some aspects. We previously showed that piceatannol is more readily absorbed upon oral ingestion, has higher metabolic stability in vivo, ${ }^{15}$ and shows enhanced endothelial nitric oxide synthase ${ }^{9}$ and SIRT1 ${ }^{14}$ expression.

\footnotetext{
${ }^{a}$ Research Institute for Science and Engineering, Waseda University, 3-4-1 Okubo, Shinjuku-ku, Tokyo 169-8555, Japan. E-mail: arai@aoni.waseda.jp, ayocha@waseda.jp; Fax: +81-3-5286-8190; Tel: +81-3-5286-3100

${ }^{b}$ School of Advanced Science and Engineering, Waseda University, 3-4-1 Okubo, Shinjuku-ku, Tokyo 169-8555, Japan. E-mail: r-kataoka.s.t@fuji.waseda.jp, otsukasatoshikun@fuji.waseda.jp

${ }^{c}$ Health Science Research Center, Morinaga \& Co., Ltd, 2-1-1 Shimosueyoshi, Tsurumi-ku, Yokohama 230-8504, Japan. E-mail: h-uchida-ji@morinaga.co.jp, m-sai-ia@morinaga.co.jp,t-ito-jj@morinaga.co.jp
}<smiles>Oc1cc(O)cc(C=Cc2ccc(O)c(O)c2)c1</smiles>

Piceatannol<smiles>Oc1ccc(/C=C/c2cc(O)cc(O)c2)cc1</smiles>

Resveratrol
Fig. 1 Structures of piceatannol and resveratrol.

While the physiological effects of piceatannol have been elucidated, its effects on cellular differentiation and the difference between its effects and those of resveratrol are poorly understood. Few studies have examined the effects of piceatannol on cellular differentiation. It was reported that piceatannol and resveratrol induce the differentiation of osteoblasts by increasing bone morphogenetic protein-2 production. ${ }^{16-18}$ Kwon et al. demonstrated that piceatannol inhibited the insulin-signaling pathway and subsequent adipogenesis in the mouse preadipocyte cell line 3T3-L1 ${ }^{19}$ Similarly, resveratrol is thought to inhibit the maturation of 3T3-L1 cells through several mechanisms, including by inhibiting insulin signaling. ${ }^{20-22}$ Thus, it is likely that piceatannol and resveratrol share some common effects. It remains unclear whether piceatannol has a specific function in cellular differentiation that is not shared by resveratrol.

Resveratrol induces the differentiation of neural stem cells (NSCs) into astrocytes, ${ }^{23}$ the most common glial cells in the central nervous system. Astrocytes have various important roles, including control of blood flow, ion transportation, the 
uptake and release of neurotransmitters, as well as energy metabolism, thereby regulating brain homeostasis. ${ }^{24,25}$ In this study, we investigated whether piceatannol affects the differentiation of mouse NSCs into astrocytes and compared its effects with those of resveratrol.

\section{Materials and methods}

\subsection{Reagents}

Piceatannol and resveratrol were purchased from Tokyo Chemical Industry (Tokyo, Japan). For in vitro experiments, the reagents were dissolved in dimethyl sulfoxide (DMSO) and administered at a final DMSO concentration of less than $0.1 \%$. For ingestion into mice, the reagents were suspended in $0.5 \%$ carboxymethyl cellulose solution just prior to use.

\subsection{In vitro differentiation of astrocytes}

The differentiation of mouse embryonic stem cells (ESCs) into astrocytes was conducted as described previously, ${ }^{26}$ but with several modifications. The J1 mouse ESC line was obtained from the American Type Culture Collection (Manassas, VA) and maintained on $0.1 \%$ gelatin-coated dishes with mitomycin C-treated mouse embryonic fibroblasts (Kitayama Labs, Nagano, Japan). The medium for culturing ESCs consisted of Dulbecco's modified Eagle's medium (DMEM) (Wako Pure Chemical, Osaka, Japan) supplemented with 15\% fetal bovine serum (HyClone, Logan, UT), 1\% L-glutamine (Thermo-Fisher Scientific, Waltham, MA), 1\% penicillin-streptomycin (Thermo-Fisher Scientific), 1\% non-essential amino acids (Thermo-Fisher Scientific), 0.18\% 2-mercaptoethanol (ThermoFisher Scientific), and $1000 \mathrm{U} \mathrm{mL}^{-1}$ leukemia inhibitory factor (Merck Millipore, Billerica, MA). Embryoid bodies (EBs) were induced by the hanging drop method at 7500 cells per droplet $(20 \mu \mathrm{L})$ for $72 \mathrm{~h}$. The EBs were then cultured for 4 days in nonadhesive bacterial dishes (Thermo-Fisher Scientific) in astrocyte-conditioned medium (ACM) (Sumitomo Bakelite, Tokyo, Japan) supplemented with $20 \mathrm{ng} \mathrm{mL}^{-1}$ rhFGF-2 and $20 \mathrm{ng}$ $\mathrm{mL}^{-1}$ rhEGF (R\&D Systems, Minneapolis, MN). Next, the cells were transferred into dishes coated with Matrigel (BD Biosciences, Franklin Lakes, NJ) containing neurobasal medium (Thermo-Fisher Scientific), supplemented with $20 \mathrm{ng}$ $\mathrm{mL}^{-1}$ rhFGF-2, $20 \mathrm{ng} \mathrm{mL}^{-1}$ rhEGF, 2\% B-27 supplement (Thermo-Fisher Scientific), and $1 \%$ penicillin-streptomycin, and were incubated for 20 days. Approximately 10 days after the transfer, the NSCs migrated outside of the EBs. These NSCs were collected and cryopreserved. The cryopreserved NSCs were seeded on a Matrigel-coated 96-well plate in neurobasal medium supplemented with $20 \mathrm{ng} \mathrm{mL}^{-1}$ rhFGF-2, $20 \mathrm{ng}$ $\mathrm{mL}^{-1}$ rhEGF, 2\% B-27 supplement, and 1\% penicillin-streptomycin at 10000 cells per well and were cultured for 3 days. Finally, the cells were transferred into ACM to trigger their differentiation into astrocytes. The cells were cultured for 3 days in the presence of piceatannol, resveratrol, or DMSO (as control), and the medium was changed daily. In order to main- tain the NSC state, the cells were cultured in NSC medium for the final 3 days.

\subsection{MTT assay}

The 3-(4,5-dimethyl-2-thiazolyl)-2,5-diphenyltetrazolium bromide (MTT) assay was performed according to the standard protocol. Briefly, MTT (Tokyo Chemical Industry) solution was directly added to cells cultured in a 96-well plate. After $3 \mathrm{~h}$ of incubation, the medium was removed and the formazan product was dissolved in DMSO. Absorbance in each well was quantified with a Fusion plate reader (PerkinElmer, Waltham, MA). Nonlinear regression analysis and estimation of $\mathrm{IC}_{50}$ were performed using R software (https://www.r-project.org/).

\subsection{Immunofluorescence}

The cells were rinsed twice with phosphate-buffered saline (PBS), fixed in $4 \%$ paraformaldehyde at $4{ }^{\circ} \mathrm{C}$ for $30 \mathrm{~min}$, rinsed three times, and blocked in PBS with $5 \%$ skim milk and $0.2 \%$ Triton $\mathrm{X}-100$ at $4{ }^{\circ} \mathrm{C}$ for $30 \mathrm{~min}$. Then, they were incubated with monoclonal antibodies against glial fibrillary acidic protein (GFAP) (1:500, MAB360, Merck Millipore) or NES (1:200, ab11306, Abcam, Cambridge, UK) at $4{ }^{\circ} \mathrm{C}$ overnight. The cells were then visualized with Chromeo 488 goat antimouse IgG (1:200) (Active Motif, Carlsbad, CA), and their nuclei were counterstained with Hoechst 33342 (Dojindo Laboratories, Kumamoto, Japan) in order to enumerate whole cells. Fluorescent images were obtained with an IX71 microscope (Olympus, Tokyo, Japan) at $10 \times$ magnification.

\subsection{Flow cytometry}

The cells were dispersed in $0.25 \%$ trypsin-ethylenediaminetetraacetic acid (Thermo-Fisher Scientific) and transferred into $1.5 \mathrm{~mL}$ microcentrifuge tubes. The cells were then fixed using $4 \%$ paraformaldehyde at $4{ }^{\circ} \mathrm{C}$ for $30 \mathrm{~min}$ and rinsed with PBS twice. Next, the cells were blocked in PBS with 5\% skim milk and $0.2 \%$ Triton $\mathrm{X}-100$ at $4{ }^{\circ} \mathrm{C}$ for $30 \mathrm{~min}$. The cells were labeled in two steps: with the anti-GFAP antibody $(1: 1000)$ (MAB360, Merck Millipore) at room temperature for $2 \mathrm{~h}$ and with the Chromeo 488 goat anti-mouse IgG (1:400) (Active Motif) at $4{ }^{\circ} \mathrm{C}$ for $30 \mathrm{~min}$. The cells were then subjected to flow cytometry using Cell Sorter SH800 (Sony, Tokyo, Japan). The series of experiments was independently repeated three times to confirm reproducibility.

\section{6. qPCR analysis}

Total RNA was extracted with an RNeasy Plus Mini kit (QIAGEN, Hilden, Germany). Complementary DNA (cDNA) was synthesized from $1 \mu \mathrm{g}$ of total RNA with the oligo(dT)20 primer and using the PrimeScript II 1st strand cDNA Synthesis Kit (TaKaRa Bio, Shiga, Japan). Real-time PCR analyses were performed on a 7500 Real-Time PCR System (Applied Biosystems, Foster City, CA) using the THUNDERBIRD SYBR qPCR Mix (Toyobo, Osaka, Japan). The primer sequences were as follows: 5'-TGGTGAAGGTCGGTGTGAAC-3' and 5'-AATGAAGGGGTCGTTGATGG-3' for Gapdh, 5'-GCAACCGGGTCAAGTTGGT-3' and 5'-GTCGTTGGAGTAGTTGGGGG-3' for Mash1, 
5'-GATGACGATGACAGAACGTCACA-3' and 5'-GGATCGGTGCCAATCATGAG-3' for Sirt1, 5'-CCATTCCTGTACAGACTTTCTCCAA-3' and 5'-GGCCTTCTGACACGGATTTG-3' for Gfap, and 5'-AACGACAAAATACCTGTGGCCTTG-3' and 5'CTCCACTTTGCTCTTGACTTCTATCTTG-3' for $I l 1 b$. A series of experiments, including differentiation, RNA extraction, cDNA synthesis, and qPCR, were independently performed three times, and representative results are shown.

\subsection{Western blotting}

Whole protein of astrocytes was extracted with a sodium dodecyl sulfate (SDS) sample buffer, boiled at $100{ }^{\circ} \mathrm{C}$ for $5 \mathrm{~min}$, separated by SDS-polyacrylamide gel electrophoresis (SDS-PAGE), and transferred onto an Immobilon $\mathrm{P}$ polyvinylidene fluoride (PVDF) membrane (Merck Millipore). The blot was blocked in $10 \%$ skim milk in $0.1 \%$ Tween/PBS for $60 \mathrm{~min}$, followed by incubation at $4{ }^{\circ} \mathrm{C}$ overnight with the following primary antibodies: anti-MASH1 (1:100, 24B72D11, eBioscience, San Diego, CA), anti-SIRT1 (1:200, D60E1, Cell Signaling Technology, Danvers, MA), and anti-ACTB $(1: 2000$, ab6276, Abcam). After being washed with $0.1 \%$ Tween/ PBS six times, the blot was incubated at room temperature for $2 \mathrm{~h}$ with horseradish peroxidase (HRP)-conjugated secondary antibodies (GE Healthcare, Chicago, IL). Signals were developed with Super Signal West Pico Chemiluminescent Substrate (Thermo-Fisher Scientific) for SIRT1 and ACTB or Super Signal West Femto Maximum Sensitivity Substrate (Thermo-Fisher Scientific) for MASH1, and detected with a Luminescent Image Analyzer LAS-3000 (Fuji Film, Tokyo, Japan).

\subsection{Animal experiments}

All experiments were approved by the Committee for Animal Experiments of Waseda University (2015-A067) and performed in accordance with the Guidelines for Proper Conduct of Animal Experiments (Science Council of Japan, 2006). Eightweek-old male BALB/cByJJcl mice were obtained from Clea Japan, Inc. (Tokyo, Japan). The mice were maintained under standard conditions (12-12 h light/dark cycle at $25{ }^{\circ} \mathrm{C}$, free access to normal rodent chow (CE-2, Clea Japan) and water) and acclimated to the laboratory conditions for 1 week before experiments. Piceatannol or resveratrol was administered at a volume of $8 \mathrm{~mL}$ per $\mathrm{kg}$ body weight (BW) by gavage once per day for 2 weeks. The mice were killed on the day after the final administration. For qPCR analyses, the mice were killed by cervical dislocation. Next, the hippocampus was surgically isolated from the brain, snap-frozen immediately in liquid nitrogen, and stored at $-80{ }^{\circ} \mathrm{C}$. After cryogenic grinding, the tissues were subjected to RNA extraction and the following experiment. Immunohistochemistry was conducted by Genostaff (Tokyo, Japan). Briefly, after perfusion fixation, the brains were dissected and embedded in paraffin. Serial coronal sections including those of the hippocampus were cut at $6 \mu \mathrm{m}$. For all sections, the first section in every 10 sections was stained with hematoxylin and eosin, while the second and the fourth sections in every 10 sections were stained with anti-
GFAP (Z0334, Dako, Carpinteria, CA) and anti-F4/80 (BM4008, OriGene, Rockville, MD), respectively. Images were obtained with a Leica DM 2500 microscope (Leica Microsystems, Wetzlar, Germany). The positions of the sections in the brain were determined on the basis of the hematoxylin and eosin images to avoid a positional difference between individuals. As a result, seven serial sections were selected from each individual. The number of GFAP-positive stellate cells in the dentate gyrus in each section was determined, and the area of the dentate gyrus was determined using Image software $(\mathrm{NIH}$, Bethesda, MD) to calculate the counts per area. The sum of the values for all 7 sections from one individual was regarded as the relative number of GFAP-positive cells in the dentate gyrus of that individual.

\subsection{Statistical analysis}

Student's $t$-test was used to determine the statistical significance of the observed differences. Microsoft Excel (Seattle, WA) was used for statistical calculations.

\section{Results}

We investigated the effects of piceatannol and resveratrol on astrocyte differentiation using the selective in vitro differentiation model of mouse NSCs into astrocytes. NSCs were prepared from mouse ESCs and were differentiated into astrocytes by culturing them in ACM for 3 days. Immunofluorescence using monoclonal antibodies against the astrocyte marker GFAP selectively labeled cells with a star-like morphology, a characteristic of mature astrocytes (Fig. 2A, left). Several studies have reported that GFAP is also expressed in a specific type of NSCs; ${ }^{27}$ however, no GFAP expression was detected in undifferentiated NSCs, indicating that the GFAP-positive cells in our experiments were astrocytes. Under the differentiation condition, almost no cells expressed NES, the NSC marker (Fig. 2A, right).

Initially, piceatannol and resveratrol were added to undifferentiated NSCs at several concentrations. Both compounds exhibited cytotoxicity against NSCs at higher concentrations (piceatannol, $\mathrm{IC}_{50}=13.5 \mu \mathrm{M}$; resveratrol, $\mathrm{IC}_{50}=9.2 \mu \mathrm{M}$ ), while no effect was observed at a concentration of $2.5 \mu \mathrm{M}$ or less (Fig. 2B). Similarly, when piceatannol and resveratrol were added during differentiation into astrocytes, the number of living cells was significantly reduced at a concentration of $5 \mu \mathrm{M}$, but not at $2.5 \mu \mathrm{M}$ (Fig. 2C). Therefore, we induced differentiation at lower concentrations $(2.5$ and $1 \mu \mathrm{M})$. We counted the number of total cells (visualized using Hoechst 33342) and astrocytes (i.e., GFAP-positive star-shaped cells) using fluorescent microscopy to estimate the astrocyte differentiation rate. Treatment with $2.5 \mu \mathrm{M}$ piceatannol significantly increased the number of astrocytes $(18 \pm 16 \%$ increase compared with that in control, $p=0.045)$. Surprisingly, resveratrol did not affect astrocyte differentiation at either concentration (Fig. 2D). In addition, differentiated cells treated with $2.5 \mu \mathrm{M}$ piceatannol or resveratrol were fixed, incubated with the GFAP 
A

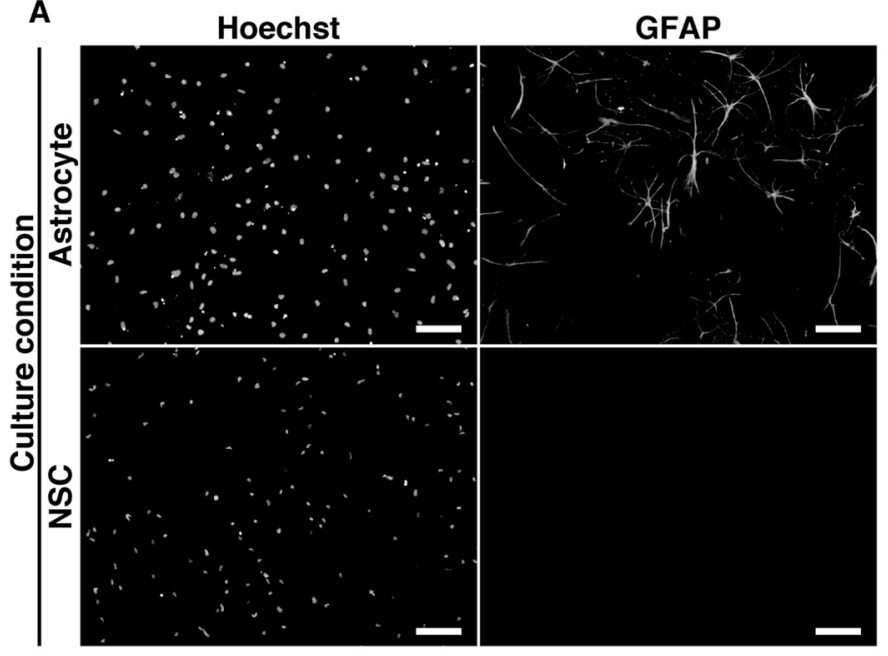

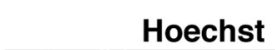

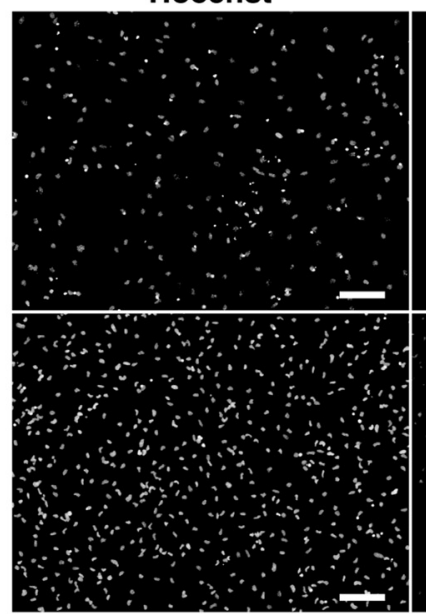

NES

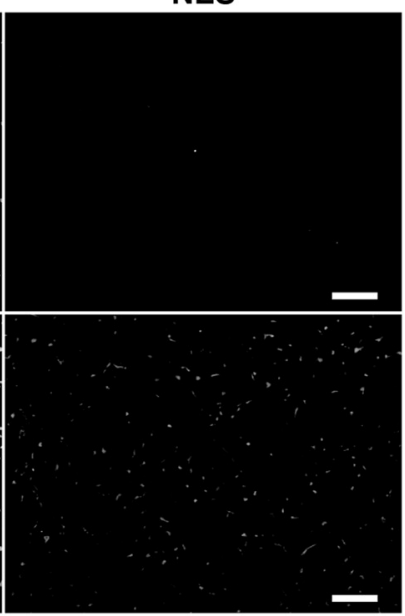

B

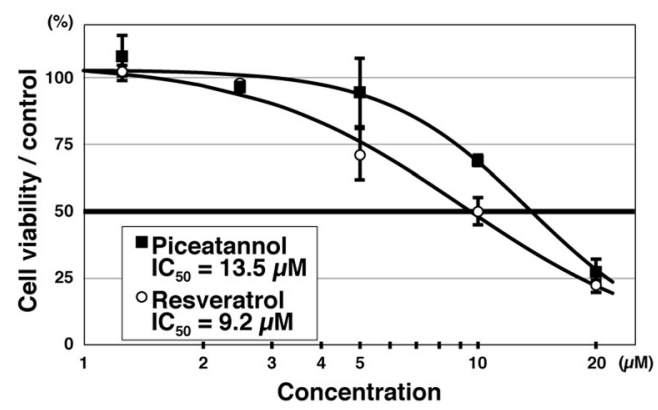

E
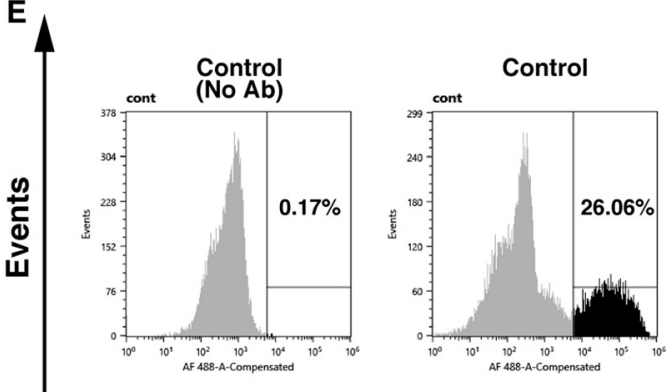

\section{C}

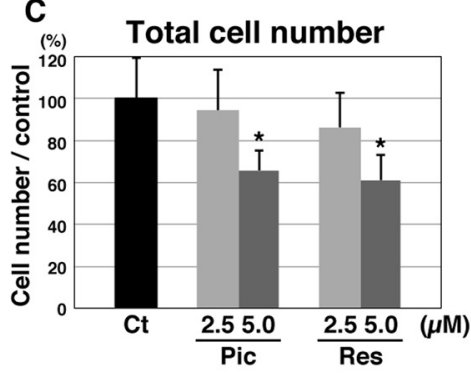

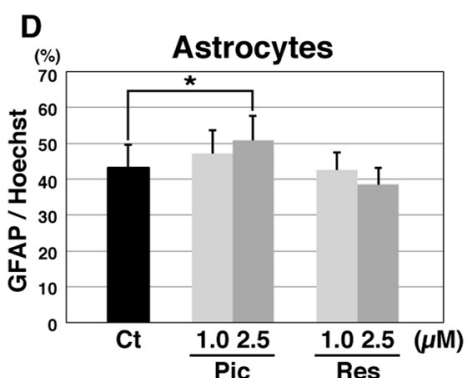
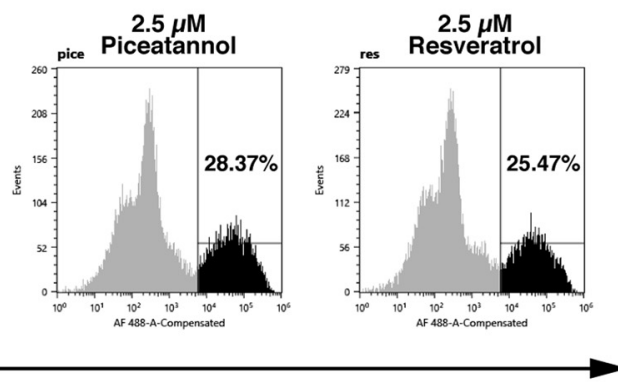

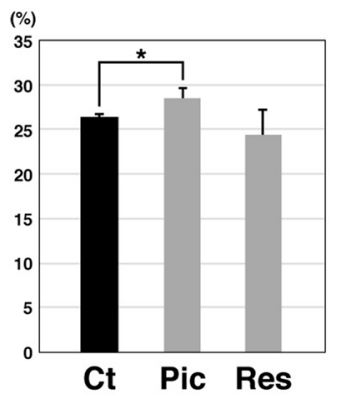

Fig. 2 Piceatannol induced differentiation of neural stem cells (NSCs) into astrocytes. (A) Fluorescence imaging of NSCs differentiated into astrocytes (upper panel) and undifferentiated NSCs (bottom panel). Nuclei of whole cells were stained with Hoechst 33342, and the astrocytes and NSCs were visualized using an antibody against glial fibrillary acidic protein (GFAP) or NES, respectively. Scale bar, $100 \mu \mathrm{m}$. (B) Viability of undifferentiated NSCs treated with piceatannol or resveratrol at various concentrations for 3 days. (C, D) NSCs were differentiated into astrocytes in the presence of dimethyl sulfoxide (DMSO) (control, Ct), piceatannol (Pic), or resveratrol (Res) at the indicated concentrations. The number of whole cells, estimated by Hoechst 33342 staining and expressed as the percentage of the control, and the rates of GFAP-positive astrocytes, i.e., the percentage of the whole cell number in each condition, are shown in (C) and (D), respectively. The data represent the average \pm standard deviation of 3 wells (C) or 8 wells (D). Asterisk indicates the statistical significance in a Student's $t$-test $(p<0.05)$. (E) Flow cytometry analysis of piceatannol- and resveratroltreated cells. Representative histograms from three independent experiments are shown. The graph depicts the average \pm standard deviation of the rates of astrocytes. Asterisk, $p=0.041$.

antibody, and analyzed using flow cytometry. In accordance with the results shown above, the number of GFAP-positive cells increased with the addition of $2.5 \mu \mathrm{M}$ piceatannol $(8 \pm$ $4 \%$ increase compared with that in control, $p=0.041$ ) but not by resveratrol (Fig. 2E).
A previous study suggested that resveratrol leads to the activation of SIRT1 and subsequent repression of the transcription of the Mash1 gene, a target of SIRT1 histone deacetylation. Because MASH1 is a transcription factor that prevents astrocyte differentiation, ${ }^{23}$ repression of Mash1 by resveratrol was 
A

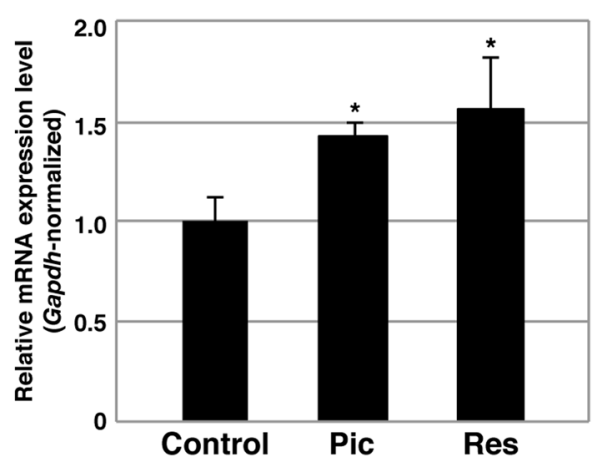

B

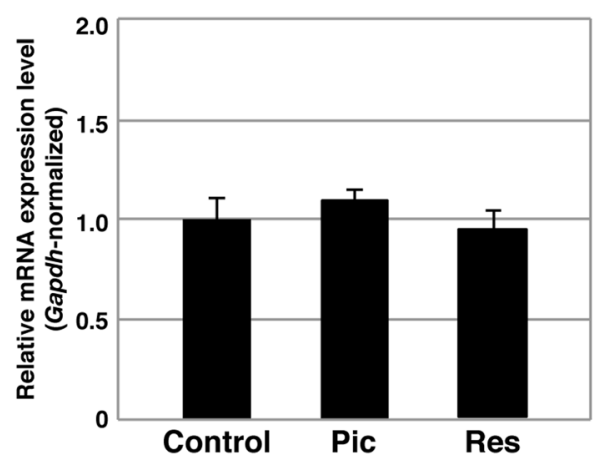

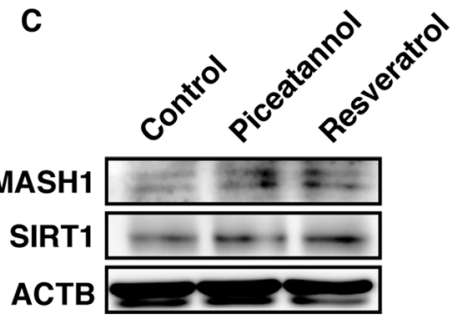

Fig. 3 Expression analysis of Mash1 and Sirt1 in the differentiated cells. (A, B) Relative expression levels of Mash1 (A) and Sirt1 (B) in differentiated cells treated with $2.5 \mu \mathrm{M}$ piceatannol (Pic) or $2.5 \mu \mathrm{M}$ resveratrol (Res). The graph depicts the average \pm standard deviation of triplicate qPCR samples normalized by Gapdh. Asterisk indicates the statistical significance in a Student's $t$-test $(p<0.05)$. (C) Protein levels of MASH1 and SIRT1 in differentiated cells treated with $2.5 \mu \mathrm{M}$ piceatannol or $2.5 \mu \mathrm{M}$ resveratrol. ACTB served as an internal control.

believed to induce astrocyte differentiation. Because piceatannol, which is similar to resveratrol, is known to activate SIRT1, we investigated whether piceatannol is involved in the silencing of Mash1. NSCs were differentiated into astrocytes by piceatannol and resveratrol at $2.5 \mu \mathrm{M}$, and the cells were subsequently harvested and subjected to qPCR analysis. The expression levels of Mash1 were not decreased, but rather slightly increased, in both cases (Fig. 3A). Thus, in contrast to a previous report, neither piceatannol nor resveratrol repressed the transcription of Mash1 under our experimental conditions. This previous study also showed that pro-oxidative conditions stimulate mRNA expression of Sirt1, thereby promoting astrocyte differentiation. ${ }^{23}$ We confirmed that neither piceatannol nor resveratrol altered the expression levels of Sirt1 in astrocytes at $2.5 \mu \mathrm{M}$ (Fig. 3B). In addition, MASH1 and SIRT1 were substantially not affected at the protein level (Fig. 3C).

We investigated whether piceatannol promotes the differentiation of the NSCs into astrocytes in the brains of adult mice. We focused on the hippocampus, where adult NSCs reside. Piceatannol was administered orally to adult mice at doses of 3 , 10 , or $30 \mathrm{mg}$ per $\mathrm{kg} \mathrm{BW}$ for 2 weeks, and then the hippocampus of the mice was surgically isolated from the brain. The mRNA expression levels of Gfap in the hippocampus were determined by qPCR analysis. Administration of $10 \mathrm{mg}$ per $\mathrm{kg}$ BW piceatannol significantly increased the level of Gfap expression in the hippocampus compared with that in vehicleadministered mice (Fig. 4A, $p=0.015$ ). Administration of $30 \mathrm{mg}$ per $\mathrm{kg}$ BW piceatannol also increased the Gfap level, and the effect was slightly lower than that of $10 \mathrm{mg}$ per $\mathrm{kg}$ BW, although these differences were not statistically significant ( $p=0.064$ versus control and $p=0.246$ versus $10 \mathrm{mg}$ per $\mathrm{kg} \mathrm{BW}$, respectively). We could not determine whether an excess of piceatannol exerted the opposite effect on NSCs, or if this was simply due to experimental errors. Expression of Mash1 was not substantially affected in any condition (Fig. 4B). These results suggest that astrocytes were increased by the oral administration of piceatannol. The increase in astrocytes in the hippocampus was also verified by immunohistochemistry. Piceatannol or resveratrol was administered at a dose of $10 \mathrm{mg}$ per $\mathrm{kg}$ BW for 2 weeks, and coronal sections of the brain were immunostained with the GFAP antibody to count the number of astrocytes (GFAP-positive star-shaped cells) in the dentate gyrus. We confirmed that the number of astrocytes in the dentate gyrus was increased by piceatannol $(23 \pm 19 \%$ increase compared to that in control, $p=0.050$ ), but not by resveratrol (Fig. 4C and D).

It is widely known that CNS damage leads to the activation of astrocytes and subsequent upregulation of GFAP, as a process of inflammation. ${ }^{28}$ Thus, we determined whether the increase of GFAP-positive cells was associated with inflammation. Upon CNS damage, reactive microglia accumulate in the damaged site. ${ }^{29}$ We examined the expression of $\mathrm{F} 4 / 80$, a marker protein for microglia, and found that there were a few F4/80-positive cells in the median eminence, where the microglia are associated with synaptic remodeling, ${ }^{30}$ whereas no F4/ 80-positive cells were observed in the hippocampus of control mice, as well as those of mice administered piceatannol or resveratrol at $10 \mathrm{mg}$ per $\mathrm{kg}$ BW (Fig. 5A). We also examined the expression of $I l 1 b$, a gene encoding a precursor of inflammatory cytokine, because mRNA expression of this gene is known to be induced in response to proinflammatory signals. ${ }^{31}$ Expression levels of $I l 1 b$ were not substantially changed in the hippocampus of piceatannol-administered mice at doses of 3 , 10 , or $30 \mathrm{mg}$ per $\mathrm{kg} \mathrm{BW}$, compared with that of control mice (Fig. 5B). Altogether, we conclude that orally administered piceatannol did not induce inflammation in the hippocampus, and that the increase of the astrocytes was not related to inflammation.

\section{Discussion}

In this study, we explored the functional differences between piceatannol and resveratrol in astrocyte differentiation and 
A

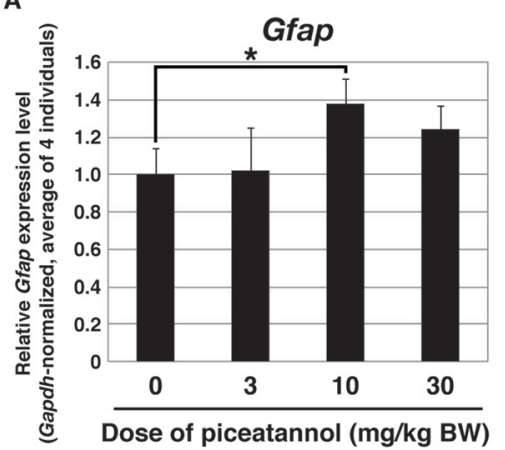

B

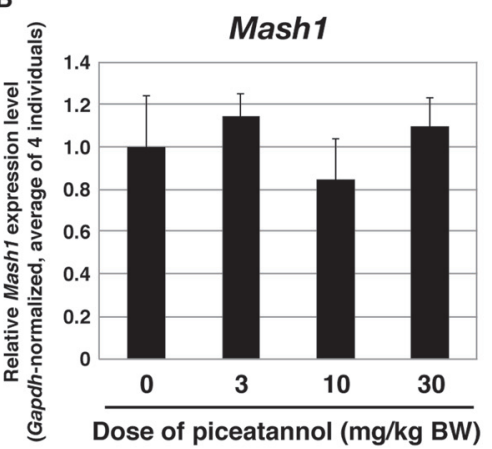

C GFAP / Hematoxylin
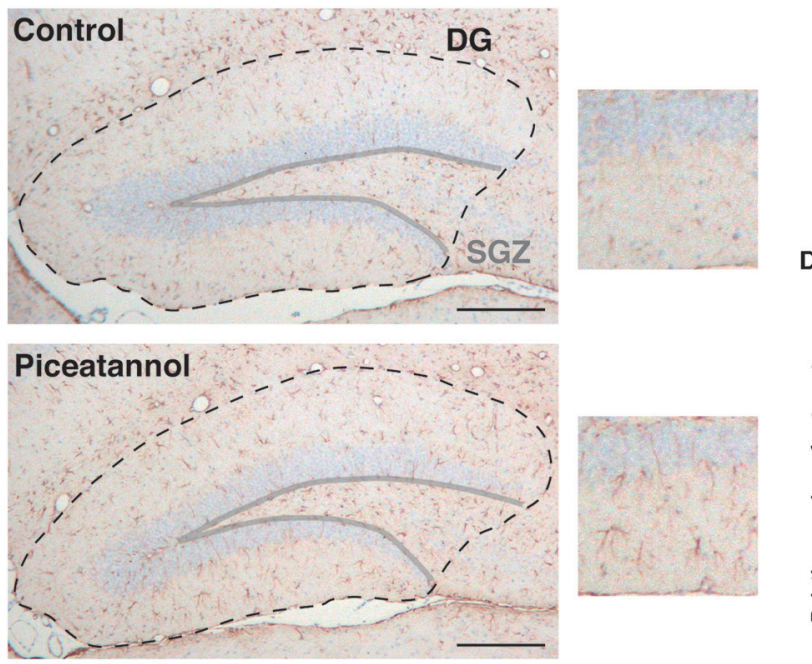

D
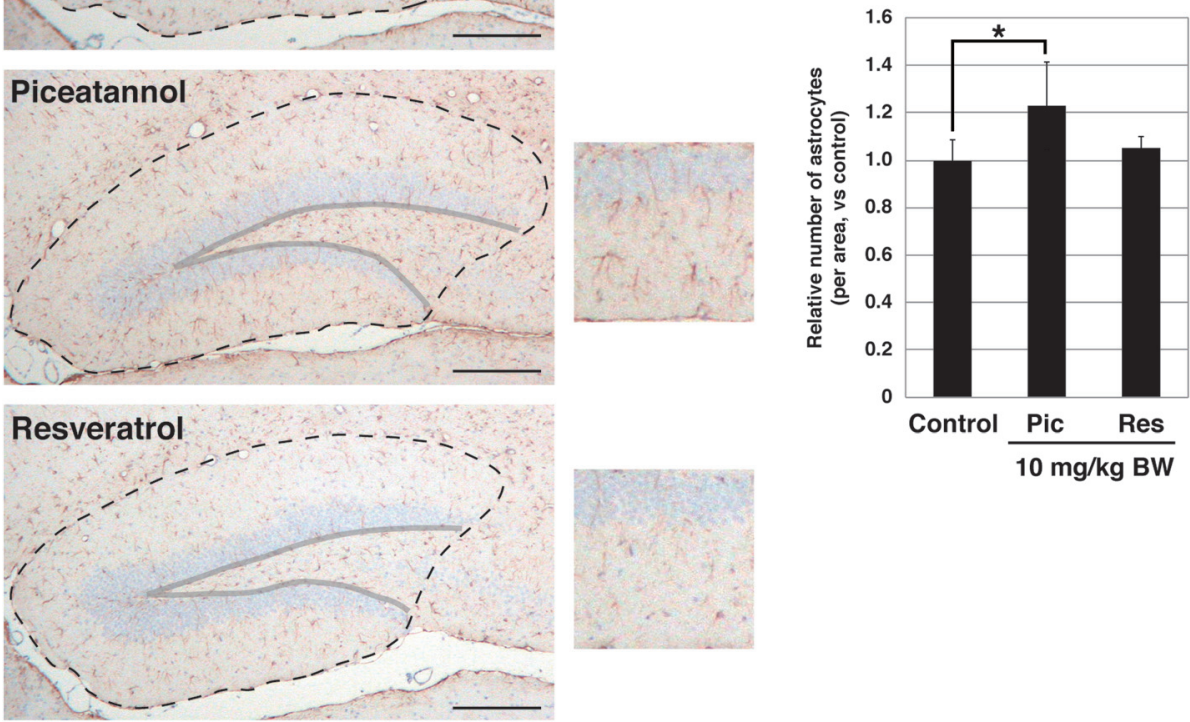

Fig. 4 Increase of astrocytes in the mouse hippocampus upon piceatannol administration. (A, B) Relative expression levels of Gfap (A) and Mash1 (B) in the hippocampus of mice administered the indicated doses of piceatannol for 2 weeks. For each individual, the average of triplicate qPCR (normalized by Gapdh) was determined. The graph depicts the average \pm standard deviation of the Gfap level of 4 individuals. Asterisk indicates the statistical significance in a Student's t-test $(p=0.015)$. (C, D) Immunohistochemistry of GFAP in the dentate gyrus (DG) of mice administered 10 mg per kg BW piceatannol or resveratrol. Representative images and their magnified views (right) are shown in (C). GFAP was stained brown, and nuclei were counterstained with hematoxylin in blue. Regions of the DG are surrounded by a dotted line. Scale bar, $200 \mu \mathrm{m}$. The average \pm standard deviation of the number of GFAP-positive stellate cells per area of 4 individuals is shown in (D). Asterisk, $p=0.050$. Note that GFAP-positive radial cells in the subgranular zone (SGZ) were not counted, as they were likely immature cells that can give rise to both neurons and astrocytes. ${ }^{27}$

found that only piceatannol promoted the differentiation of NSCs into astrocytes in vitro. The increase was relatively moderate $(8-18 \%)$, possibly due to the short-term treatment (72 hours). We also showed that 14 days of oral administration of piceatannol, but not that of resveratrol, increased the number of astrocytes in the hippocampus of adult mice. This is the first report revealing an effect of piceatannol on cellular differentiation that is not shared with resveratrol.
In contrast to a previous report, ${ }^{23}$ we observed no positive effect of resveratrol on astrocyte differentiation. Prozorovski et al. exposed mouse fetal NSCs to resveratrol at a concentration of $5 \mu \mathrm{M}$. Another group showed that resveratrol was toxic to rat fetal NSCs at $10 \mu \mathrm{M}$. They also reported that $3 \mu \mathrm{M}$ resveratrol was neither toxic nor stimulating for astrocyte differentiation. $^{32}$ Our results were consistent with the latter case, i.e., resveratrol was toxic to NSCs at the higher concen- 
A F4/80 / Hematoxylin

\section{Dentate gyrus}
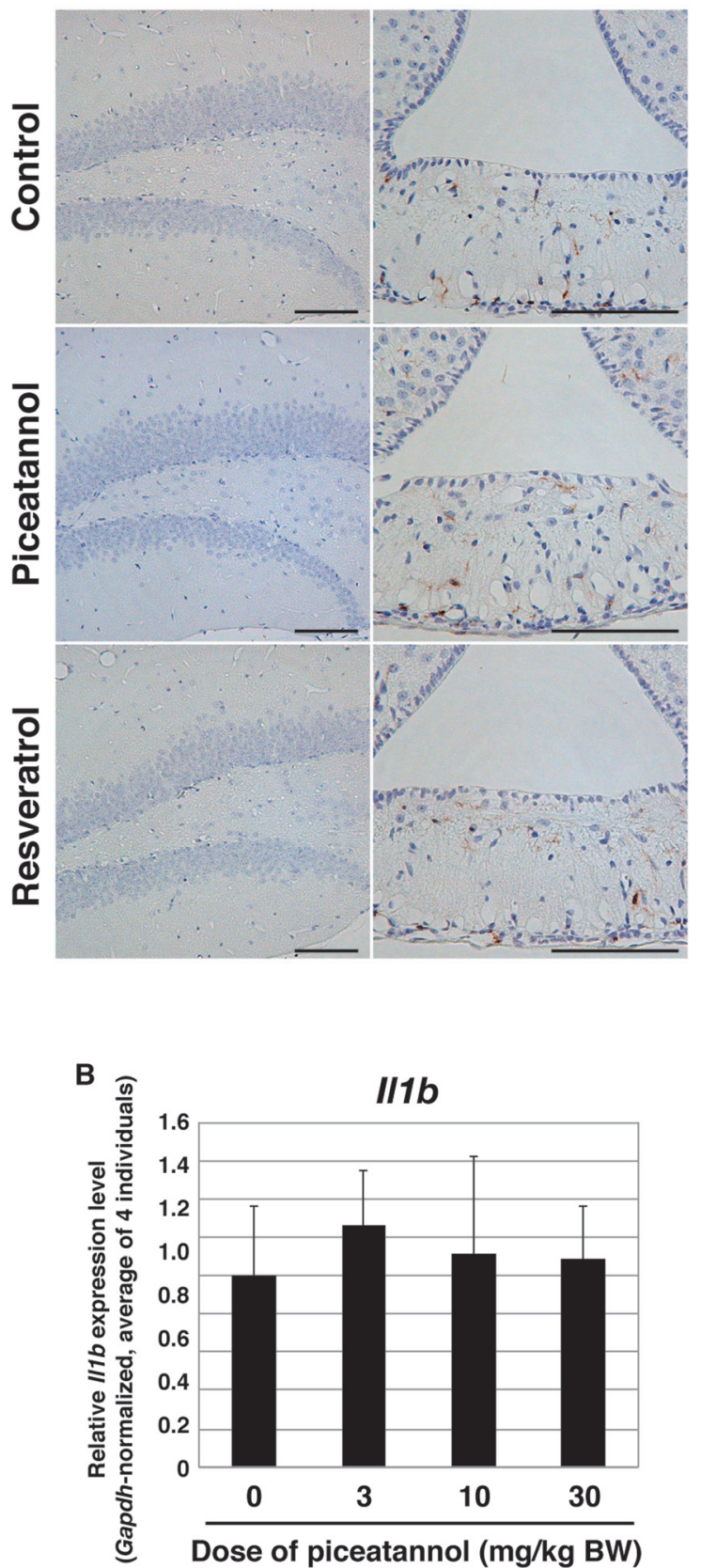

Fig. 5 Inflammation did not occur in the hippocampus of piceatannoladministered mouse. (A) Immunohistochemistry of F4/80 in the dentate gyrus (DG) and median eminence of mice administered $10 \mathrm{mg} \mathrm{kg}^{-1}$ of piceatannol or resveratrol. F4/80 was stained brown, and nuclei were counterstained with hematoxylin in blue. Note that photographs of the DG and median eminence of each were obtained from the same section, which is adjacent to the GFAP-stained section shown in Fig. 4C. (B) Relative expression levels of $111 \mathrm{~b}$ in the hippocampus of mice administered the indicated doses of piceatannol for 2 weeks. For each individual, the average of triplicate GPCR (normalized by Gapdh) was determined. The graph depicts the average \pm standard deviation of the ll1b level of 4 individuals. tration $(5 \mu \mathrm{M})$, whereas it had no effect on differentiation at the lower concentration $(2.5 \mu \mathrm{M})$. The decrease in the cell number caused by the higher concentration of resveratrol may indirectly influence astrocyte differentiation because the efficiency of differentiation is generally dependent on the cell density. Another possibility is that resveratrol induces upregulation of SIRT1 via a cell-damage pathway. The SIRT1 gene is known to be transcriptionally upregulated by E2F1 upon DNA damage caused by etoposide treatment, to suppress apoptosis. $^{33}$ In addition, recent studies showed that at a concentration of $10 \mu \mathrm{M}$ or more, resveratrol induced DNA doublestrand breaks in normal human cells as well as in tumor cells. ${ }^{34,35}$ Thus, excess resveratrol may induce the expression of SIRT1 by causing DNA damage, thereby repressing the Mash1 gene and facilitating astrocyte differentiation. Importantly, piceatannol promoted astrocyte differentiation at a non-toxic concentration, without the upregulation of Sirt1 or downregulation of Mash1. Piceatannol also did not affect the expression level of Mash1 in the hippocampus, whereas the number of astrocytes increased. This is likely because of different mechanisms, as proposed by Prozorovski et al.; this is important when evaluating its future in vivo applications.

When an interesting effect of a compound is discovered in cultured cells, its effective dose and physiological relevance should be carefully examined in vitro. As described above, several groups showed that both piceatannol and resveratrol inhibit the adipogenesis of 3T3-L1 cells. ${ }^{19-22}$ However, their effective concentrations were $20 \mu \mathrm{M}$ or greater, which is extremely high compared to the reported maximum plasma concentration in humans after oral intake of $5 \mathrm{~g}$ resveratrol. ${ }^{36} \mathrm{In}$ contrast to these reports, Hu et al. showed that the physiologically achievable level of resveratrol $(1$ and $10 \mu \mathrm{M})$ enhanced the adipogenesis of 3T3-L1 cells. ${ }^{37}$ These opposite effects may occur because both resveratrol and piceatannol act on multiple target proteins. Piceatannol is also known as a JAK1/STAT3 inhibitor $^{38}$ and STAT3 is essential for astrocyte differentiation, ${ }^{39}$ leading to the hypothesis that piceatannol impedes astrocyte differentiation. However, in a previous study, piceatannol was used at $100 \mu \mathrm{M}$ to diminish the phosphorylation of STAT3 in cultured cells, and phosphorylation was not affected at a concentration of $10 \mu \mathrm{M}$. Given that the maximum plasma concentration of piceatannol in rats was no more than $8 \mu \mathrm{M}$ after oral administration of $88 \mathrm{mg} \mathrm{kg}^{-1}$ of BW, ${ }^{15}$ orally ingested piceatannol is likely not sufficient for modulating astrocyte differentiation through the JAK1/STAT3 pathway. Although it remains unknown whether piceatannol can pass through the blood-brain barrier, the effective dose of piceatannol for promoting astrocyte differentiation $(2.5 \mu \mathrm{M})$ was within the range of its possible plasma concentration. When administered at a physiologically achievable dose, piceatannol did not inhibit the JAK1/STAT3 pathway, but induced astrocyte differentiation through another pathway that is more sensitive to piceatannol.

Currently, the molecular mechanism by which piceatannol stimulates astrocyte differentiation remains unclear, but the responsible target molecule appears to be regulated only by piceatannol and not by resveratrol. Our results suggest that 
this activity is not mediated by SIRT1, a common target of piceatannol and resveratrol. The precise mechanism, particularly the main target of piceatannol in the induction of astrocyte differentiation, requires further analysis.

Recent increasing evidence suggests that astrocyte impairment is associated with mental illness. ${ }^{40}$ For example, pharmacological lesions of glial cells in the prefrontal cortex of mice caused depression-like behavior, suggesting that a numerical decrease in astrocytes is one of the etiologies of depression. ${ }^{41}$ Another study showed that astrocyte function in the hippocampus is required for recovery from depression by pharmacotherapy in rats. ${ }^{42}$ Although there are many other factors participating in the onset or exacerbation of depression and mental stress-associated diseases, these reports prompt us to speculate that an increase in astrocytes might be one way to prevent and/or support recovery from these diseases. In this study, we demonstrated that orally administered piceatannol increased astrocytes in the adult hippocampus, implying that piceatannol has a beneficial effect against mental illness. On the basis of the results of our in vitro studies, the intake of piceatannol may result in the differentiation of hippocampal NSCs into astrocytes in vivo. If this is the case, however, whether increasing astrocytes at the expense of the NSCs is beneficial or not should be carefully considered, because the number of adult NSCs, which is also required for neurogenesis, is limited. ${ }^{43}$ In future studies, confirming the antistress effects of piceatannol in animals, especially in an astrocyte-impaired model, will solve this question.

In conclusion, piceatannol promoted the differentiation of NSCs into astrocytes at a low, non-toxic, and physiologically achievable dose in vitro, and is likely effective in vivo. This function is not shared by resveratrol, thus, highlighting a novel beneficial effect of piceatannol.

\section{Conflict of interest}

The authors declare no conflict of interest.

\section{Acknowledgements}

The SIRT1 antibody was kindly provided by Drs Hirosawa and Hayakawa. This work was funded by the Adaptable and Seamless Technology Transfer Program through target-driven R\&D (AS262Z02620N to DA) from the Japan Science and Technology Agency, and the Research Institute for Science and Engineering, Waseda University.

\section{Notes and references}

1 Y. Matsui, K. Sugiyama, M. Kamei, T. Takahashi, T. Suzuki, Y. Katagata and T. Ito, Extract of passion fruit (Passiflora edulis) seed containing high amounts of piceatannol inhibits melanogenesis and promotes collagen synthesis, J. Agric. Food Chem., 2010, 58, 11112-11118.
2 H. Piotrowska, M. Kucinska and M. Murias, Biological activity of piceatannol: leaving the shadow of resveratrol, Mutat. Res., 2012, 750, 60-82.

3 K. Ashikawa, S. Majumdar, S. Banerjee, A. C. Bharti, S. Shishodia and B. B. Aggarwal, Piceatannol inhibits TNFinduced NF-kappaB activation and NF-kappaB-mediated gene expression through suppression of IkappaBalpha kinase and p65 phosphorylation, J. Immunol., 2002, 169, 6490-6497.

4 M. Murias, W. Jäger, N. Handler, T. Erker, Z. Horvath, T. Szekeres, H. Nohl and L. Gille, Antioxidant, prooxidant and cytotoxic activity of hydroxylated resveratrol analogues: structure-activity relationship, Biochem. Pharmacol., 2005, 69, 903-912.

5 M. Lagouge, C. Argmann, Z. Gerhart-Hines, H. Meziane, C. Lerin, F. Daussin, N. Messadeq, J. Milne, P. Lambert, P. Elliott, B. Geny, M. Laakso, P. Puigserver and J. Auwerx, Resveratrol improves mitochondrial function and protects against metabolic disease by activating SIRT1 and PGC-1alpha, Cell, 2006, 127, 1109-1122.

6 K. J. Pearson, J. A. Baur, K. N. Lewis, L. Peshkin, N. L. Price, N. Labinskyy, W. R. Swindell, D. Kamara, R. K. Minor, E. Perez, H. A. Jamieson, Y. Zhang, S. R. Dunn, K. Sharma, N. Pleshko, L. A. Woollett, A. Csiszar, Y. Ikeno, D. Le Couteur, P. J. Elliott, K. G. Becker, P. Navas, D. K. Ingram, N. S. Wolf, Z. Ungvari, D. A. Sinclair and R. de Cabo, Resveratrol delays age-related deterioration and mimics transcriptional aspects of dietary restriction without extending life span, Cell Metab., 2008, 8, 157-168.

7 S. Kim, Y. Jin, Y. Choi and T. Park, Resveratrol exerts antiobesity effects via mechanisms involving down-regulation of adipogenic and inflammatory processes in mice, Biochem. Pharmacol., 2011, 81, 1343-1351.

8 S. Sano, K. Sugiyama, T. Ito, Y. Katano and A. Ishihata, Identification of the strong vasorelaxing substance scirpusin B, a dimer of piceatannol, from passion fruit (Passiflora edulis) seeds, J. Agric. Food Chem., 2011, 59, 6209-6213.

9 Y. Kinoshita, S. Kawakami, K. Yanae, S. Sano, H. Uchida, $\mathrm{H}$. Inagaki and T. Ito, Effect of long-term piceatannol treatment on eNOS levels in cultured endothelial cells, Biochem. Biophys. Res. Commun., 2013, 430, 1164-1168.

10 H. Uchida-Maruki, H. Inagaki, R. Ito, I. Kurita, M. Sai and T. Ito, Piceatannol lowers the blood glucose level in diabetic mice, Biol. Pharm. Bull., 2015, 38, 629-633.

11 T. Kahyo, S. Ichikawa, T. Hatanaka, M. K. Yamada and M. Setou, A novel chalcone polyphenol inhibits the deacetylase activity of SIRT1 and cell growth in HEK293T cells, J. Pharmacol. Sci., 2008, 108, 364-371.

12 G. L. Wang, Y. C. Fu, W. C. Xu, Y. Q. Feng, S. R. Fang and X. H. Zhou, Resveratrol inhibits the expression of SREBP1 in cell model of steatosis via Sirt1-FOXO1 signaling pathway, Biochem. Biophys. Res. Commun., 2009, 380, 644649.

13 J. M. Andrade, A. C. Frade, J. B. Guimarães, K. M. Freitas, M. T. Lopes, A. L. Guimarães, A. M. de Paula, C. C. Coimbra and S. H. Santos, Resveratrol increases 
brown adipose tissue thermogenesis markers by increasing SIRT1 and energy expenditure and decreasing fat accumulation in adipose tissue of mice fed a standard diet, Eur. J. Nutr., 2014, 53, 1503-1510.

14 S. Kawakami, Y. Kinoshita, H. Maruki-Uchida, K. Yanae, M. Sai and T. Ito, Piceatannol and its metabolite, isorhapontigenin, induce SIRT1 expression in THP-1 human monocytic cell line, Nutrients, 2014, 6, 4794-4804.

15 Y. Setoguchi, Y. Oritani, R. Ito, H. Inagaki, H. MarukiUchida, T. Ichiyanagi and T. Ito, Absorption and metabolism of piceatannol in rats, J. Agric. Food Chem., 2014, 62, 2541-2548.

16 K. Mizutani, K. Ikeda, Y. Kawai and Y. Yamori, Resveratrol stimulates the proliferation and differentiation of osteoblastic MC3T3-E1 cells, Biochem. Biophys. Res. Commun., 1998, 253, 859-863.

17 J. K. Chang, Y. L. Hsu, I. C. Teng and P. L. Kuo, Piceatannol stimulates osteoblast differentiation that may be mediated by increased bone morphogenetic protein-2 production, Eur. J. Pharmacol., 2006, 551, 1-9.

18 J. L. Su, C. Y. Yang, M. Zhao, M. L. Kuo and M. L. Yen, Forkhead proteins are critical for bone morphogenetic protein-2 regulation and anti-tumor activity of resveratrol, J. Biol. Chem., 2007, 282, 19385-19398.

19 J. Y. Kwon, S. G. Seo, Y. S. Heo, S. Yue, J. X. Cheng, K. W. Lee and K. H. Kim, Piceatannol, natural polyphenolic stilbene, inhibits adipogenesis via modulation of mitotic clonal expansion and insulin receptor-dependent insulin signaling in early phase of differentiation, J. Biol. Chem., 2012, 287, 11566-11578.

20 S. Chen, X. Xiao, X. Feng, W. Li, N. Zhou, L. Zheng, Y. Sun, Z. Zhang and W. Zhu, Resveratrol induces Sirt1-dependent apoptosis in 3T3-L1 preadipocytes by activating AMPK and suppressing AKT activity and survivin expression, J. Nutr. Biochem., 2012, 23, 1100-1112.

21 M. C. Mitterberger and W. Zwerschke, Mechanisms of resveratrol-induced inhibition of clonal expansion and terminal adipogenic differentiation in 3T3-L1 preadipocytes, J. Gerontol., Ser.A, 2013, 68, 1356-1376.

$22 \mathrm{H}$. Lee and J. W. Kim, High-dose Resveratrol Inhibits Insulin Signaling Pathway in 3T3-L1 Adipocytes, J. Lifestyle Med., 2013, 3, 41-47.

23 T. Prozorovski, U. Schulze-Topphoff, R. Glumm, J. Baumgart, F. Schröter, O. Ninnemann, E. Siegert, I. Bendix, O. Brüstle, R. Nitsch, F. Zipp and O. Aktas, Sirt1 contributes critically to the redox-dependent fate of neural progenitors, Nat. Cell Biol., 2008, 10, 385-394.

24 H. K. Kimelberg and M. Nedergaard, Functions of astrocytes and their potential as therapeutic targets, Neurotherapeutics, 2010, 7, 338-353.

25 M. V. Sofroniew and H. V. Vinters, Astrocytes: biology and pathology, Acta Neuropathol., 2010, 119, 7-35.

26 T. Nakayama and N. Inoue, Neural stem sphere method: induction of neural stem cells and neurons by astrocyte- derived factors in embryonic stem cells in vitro, Methods Mol. Biol., 2006, 330, 1-13.

27 Y. Liu, T. Namba, J. Liu, R. Suzuki, S. Shioda and T. Seki, Glial fibrillary acidic protein-expressing neural progenitors give rise to immature neurons via early intermediate progenitors expressing both glial fibrillary acidic protein and neuronal markers in the adult hippocampus, Neuroscience, 2010, 166, 241-251.

28 L. F. Eng and R. S. Ghirnikar, GFAP and astrogliosis, Brain Pathol., 1994, 4, 229-237.

29 H. Kettenmann, U. K. Hanisch, M. Noda and A. Verkhratsky, Physiology of microglia, Physiol. Rev., 2011, 91, 461-553.

30 C. O. Okere and H. Kaba, Heterogenous immunohistochemical expression of microglia-specific ionized calcium binding adaptor protein (Iba1) in the mouse olfactory bulb, Brain Res., 2000, 877, 85-90.

31 I. M. Adcock, Transcription factors as activators of gene transcription: AP-1 and NF-kappa B, Monaldi Arch. Chest Dis., 1997, 52, 178-186.

32 K. Wallenborg, P. Vlachos, S. Eriksson, L. Huijbregts, E. S. Arnér, B. Joseph and O. Hermanson, Red wine triggers cell death and thioredoxin reductase inhibition: effects beyond resveratrol and SIRT1, Exp. Cell Res., 2009, 315, 1360-1371.

33 C. Wang, L. Chen, X. Hou, Z. Li, N. Kabra, Y. Ma, S. Nemoto, T. Finkel, W. Gu, W. D. Cress and J. Chen, Interactions between E2F1 and SirT1 regulate apoptotic response to DNA damage, Nat. Cell Biol., 2006, 8, 10251031.

34 H. Luo, A. Yang, B. A. Schulte, M. J. Wargovich and G. Y. Wang, Resveratrol induces premature senescence in lung cancer cells via ROS-mediated DNA damage, PLOS One, 2013, 8, e60065.

35 M. Kilic Eren, A. Kilincli and Ö. Eren, Resveratrol Induced Premature Senescence Is Associated with DNA Damage Mediated SIRT1 and SIRT2 Down-Regulation, PLoS One, 2015, 10, e0124837.

36 D. J. Boocock, G. E. Faust, K. R. Patel, A. M. Schinas, V. A. Brown, M. P. Ducharme, T. D. Booth, J. A. Crowell, M. Perloff, A. J. Gescher, W. P. Steward and D. E. Brenner, Phase I dose escalation pharmacokinetic study in healthy volunteers of resveratrol, a potential cancer chemopreventive agent, Cancer Epidemiol. Biomarkers Prev., 2007, 16, 1246-1252.

37 P. Hu, L. Zhao and J. Chen, Physiologically achievable doses of resveratrol enhance 3T3-L1 adipocyte differentiation, Eur. J. Nutr., 2015, 54, 569-579.

38 L. Su and M. David, Distinct mechanisms of STAT phosphorylation via the interferon-alpha/beta receptor. Selective inhibition of STAT3 and STAT5 by piceatannol, J. Biol. Chem., 2000, 275, 12661-12666.

39 S. Hong and M. R. Song, STAT3 but not STAT1 is required for astrocyte differentiation, PLoS One, 2014, 9, e86851.

40 G. Rajkowska and C. A. Stockmeier, Astrocyte pathology in major depressive disorder: insights from human post- 
mortem brain tissue, Curr. Drug Targets, 2013, 14, 1225-1236.

41 Y. Lee, H. Son, G. Kim, S. Kim, D. H. Lee, G. S. Roh, S. S. Kang, G. J. Cho, W. S. Choi and H. J. Kim, Glutamine deficiency in the prefrontal cortex increases depressive-like behaviours in male mice, J. Psychiatry Neurosci., 2013, 38, 183-191.
42 M. Iwata, Y. Shirayama, H. Ishida, G. I. Hazama and K. Nakagome, Hippocampal astrocytes are necessary for antidepressant treatment of learned helplessness rats, Hippocampus, 2011, 21, 877-884.

43 F. H. Gage, Mammalian neural stem cells, Science, 2000, 287, 1433-1438. 